\title{
Dancing with an escape artist
}

\section{Sarah DeWeerdt describes the intricate relationship between HIV and the host immune system, each influencing the other's next moves.}

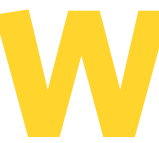

hen people talk about infectious disease, the host is often described as being at war with the pathogen, which is an apt metaphor. Look closely at HIV infection, however, and it also seems like a dance: the virus and the immune system match each other, step for step, in a performance as intricate as it is deadly.

Each person's immune system is unique and dances differently. Although HIV infection usually leads to AIDS, the timing and course of progression varies greatly from person to person.

In the past few years - thanks to methods that detect HIV soon after infection - researchers have learnt that the immune response in the first weeks often sets the long-term course of the disease. They are also learning how the body makes antibodies to HIV, and how this immune response evolves over time - insights that might aid the search for a vaccine.

HIV becomes detectable in the bloodstream about ten days after the initial infection. Over the next two to three weeks, it spreads through the body, replicating wildly. At the same time, killer T cells - which recognize and destroy infected cells - begin proliferating rapidly.

HIV levels in the bloodstream peak 21 to 28 days after infection, and T cells spike a week or two later. As T cells accumulate, they begin to reduce the amount of virus in the bloodstream.

With some viruses, this is where the dance ends. "There are many infections we have where T-cell immune responses control the virus," says Sir Andrew McMichael, professor of immunology at Oxford University's Weatherall Institute of Molecular Medicine. In these cases - chicken pox, for example - the virus remains in the body, but usually does not cause long-term problems.

In most people infected with HIV, however, killer T cells become progressively less effective. HIV has an almost limitless capacity to mutate, and in effect is constantly trying on new disguises - turning the dance into a masquerade.

Because T cells respond to HIV, it is tempting to conclude that the virus leads the dance. The immune system also exerts a subtle influence,

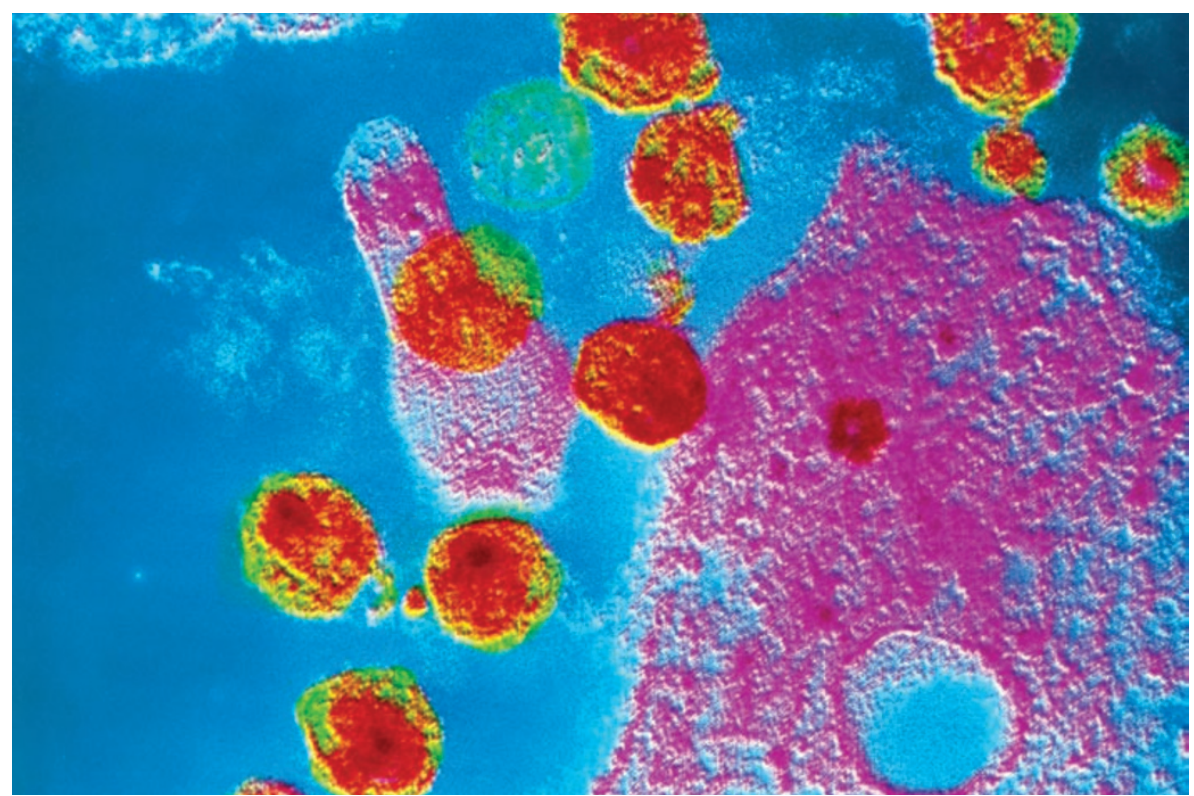

HIV (red) selectively targets and kills CD4 immune cells (pink), the master coordinators of the immune response. however, shaping the evolution of the virus. When virus levels initially peak, HIV is relatively homogeneous, with millions of identical copies of the infecting strain. Last year, researchers showed that the viral sequence later evolves precisely in the parts to which the $\mathrm{T}$ cells respond ${ }^{1}$.

"The mutants [are] being selected very rapidly, within days, right in front of your eyes," says McMichael, who led the study. Within weeks, the body is teeming with 'escape mutants' that the $\mathrm{T}$ cells must learn to recognize.

\section{Handicapped defence}

From the beginning, HIV selectively targets and kills CD4 or helper T cells, "the master coordinators of the host immune response," notes John Moore, professor of immunology and microbiology at Weill Cornell Medical College in New York. "So you have this hole in the immune repertoire."

Eventually, the depletion of CD4 cells leaves the body susceptible to pathogens, signalling the progression to AIDS. Even early on, however, the CD4 cells are dysfunctional, impairing the immune response to HIV. Without functioning helper T cells, newborn killer $\mathrm{T}$ cells fail to mature, or die prematurely, and so the body becomes progressively less able to generate new ones as the virus evolves.

Three to six months after infection, the level of HIV in the bloodstream stabilizes to a 'set point'. This predicts the course of an individual's disease: a higher set point usually means a quick progression to AIDS, and a lower set point means a slower sequence.

What defines the set point is not fully understood, but "there's some sort of balance between the nature or virulence of the virus and the immune response of the host," McMichael says. For example, a highly virulent strain of HIV is likely to result in a high set point even if the immune response is strong.

The varying strength of the immune response can be traced (see sidebar) in large part to human leukocyte antigen (HLA) molecules. Three types of HLA class I molecule, HLA-A, HLA-B and HLA-C, are found on the surface of every host cell. These HLA molecules hold on to protein scraps from the virus and signal to the killer T cells that the cell is infected.

The genes for these HLA molecules are the most variable in the genome - each has hundreds of different alleles. This variability, reshuffled each generation, allows the body to recognize a huge variety of pathogens.

The HLA-B gene seems to have the strongest overall effect on HIV, says Mary Carrington, head of the HLA Immunogenetics Section at the US National Cancer Institute. Those who carry some $H L A-B$ alleles, such as B27, B57 


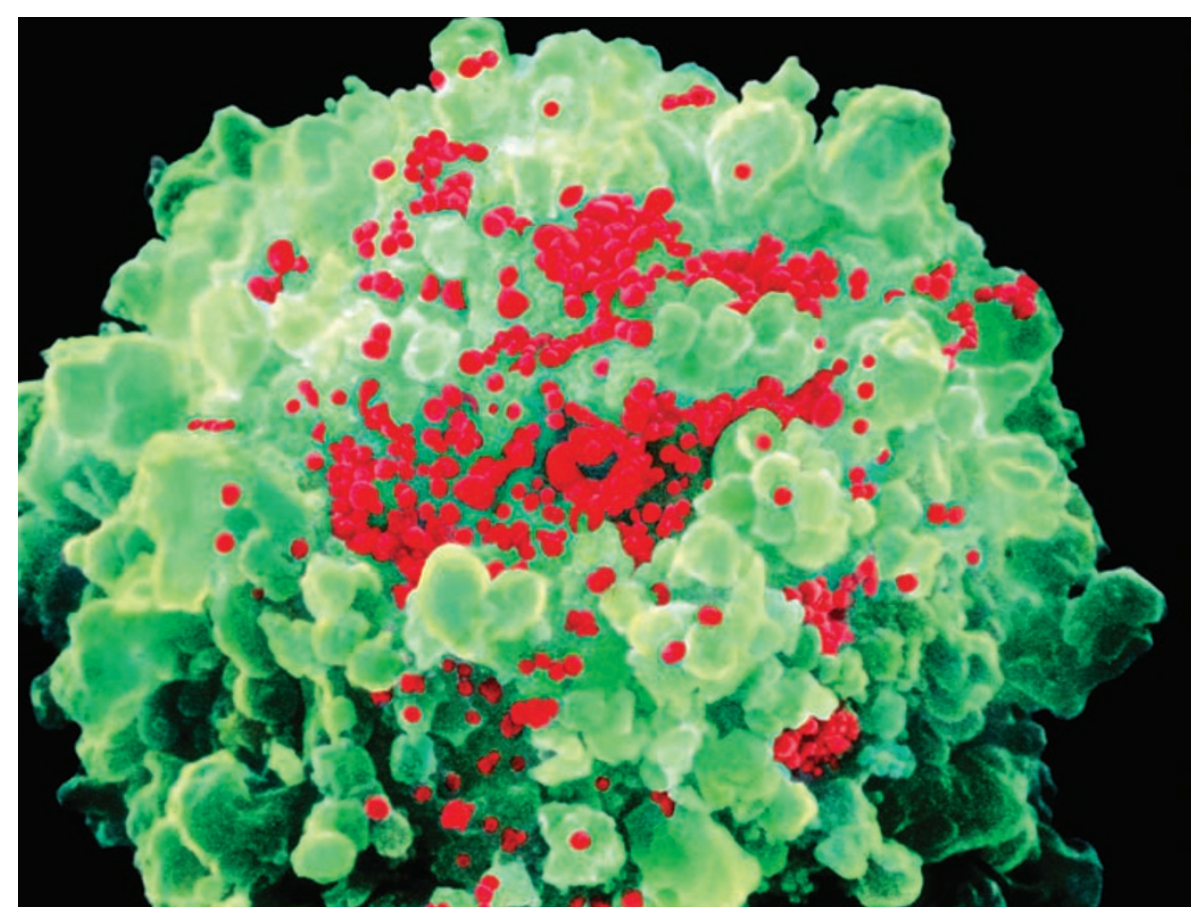

Immune cells (like the one above, in green) exert a subtle influence on the evolution of the HIV virus (red).

and $B 58$, tend to develop AIDS slowly. Other alleles, such as $B 07$ and $B 35$, are associated with a rapid progression.

Those alleles that offer protection bind to highly conserved HIV proteins that are essential to the virus' function. If the virus mutates in these proteins, it often does so at a cost, becoming less virulent - and contributing to a lower set point. In contrast, less protective alleles bind pieces of proteins that HIV "can mutate away and not have any effect on viral fitness," Carrington says.

\section{Slow burn}

The dance with HIV includes another set of partners: antibodies that recognize and bind to viral proteins. Antibodies appear early in HIV infection, at a similar time to killer T cells. The early antibodies are not effective, however, and do not reduce virus levels in the bloodstream.

Antibodies that neutralize HIV and prevent it from infecting cells are produced about three months after exposure. This is relatively late compared with the response to many viral infections, and might be partly due to viral damage to $B$ cells and the germinal centres where they mature.

These problems have long been known to occur in chronic HIV infection; however, researchers showed last year that they begin in the first weeks or even days of infection ${ }^{2}$. "It was surprising to find that there was such early destruction and damage to germinal centres," says lead investigator Barton Haynes, director of the National Institutes of Healthfunded Center for HIV/AIDS Vaccine Immunology (CHAVI).
When neutralizing antibodies finally are produced, the virus mutates to evade them, the body fashions a new response, the virus escapes again, "and so on, in an endless 'to-ing and fro-ing' between the antibody system and the virus," says Dennis Burton, professor of immunology and microbial science at the Scripps Research Institute in La Jolla, California. "But there isn't any great impact [of the antibodies] as far as we can see on the overall virus replication."

Two to three years after infection, about one in every four or five people infected with HIV begins to make broadly neutralizing antibodies, which inactivate many different strains. Ironically, by the time they make the antibodies, these people have too much virus, too many flaws in the immune response and too many reservoirs for the virus to benefit from them. What is more, HIV can mutate to evade these antibodies much faster than the body can develop new ones.

Still, broadly neutralizing antibodies are something of a 'holy grail' for vaccine researchers. "They're the sorts of antibody responses that we'd like to elicit or induce through vaccination," says Burton.

Two large-scale efforts are under way, one coordinated by CHAVI and the other by the International AIDS Vaccine Initiative, to understand better how these antibodies are made. For each effort, researchers took blood samples from hundreds of people every few months, beginning a few weeks after HIV infection.

The researchers plan to isolate the broadest, most potent antibodies from the participants, then retrace how the virus and antibody

\section{Genetic invitation}

For years, scientists have been trying to understand why some people with HIV quickly develop AIDS and others seem more resistant. One method, genomewide association, involves scanning bits of DNA scattered throughout the genome to look for variations more common in the latter group.

A handful of studies have identified several single-nucleotide polymorphisms (SNPs) associated with better control of HIV. Most variants are in human leukocyte antigen (HLA) molecules, which help the immune system to recognize infected cells.

For example, the first genome-wide association study of HIV linked a variant of the HLA-C gene to a stronger immune response and slower progression to AIDS $^{3}$. Variations in HLA-B also seem to delay disease course.

Results from an international study of about 2,000 HIV-infected individuals, expected to be published later this year, confirm the importance of the HLA system, says Mary Carrington, head of the HLA Immunogenetics Section at the US National Cancer Institute.

These studies reliably pick up common variations present in at least $4 \%$ of the population. Rarer variants might also affect HIV control, notes David Goldstein, director of the Center for Human Genome Variation at Duke University. To identify rare variants, he argues, researchers should compare whole genome sequences rather than looking at markers for differences.

Goldstein is planning to sequence the genomes of at least 50 people who have been infected with HIV for years but have not developed AIDS. He also plans to compare the genomes of people who progress relatively rapidly with those who progress slowly.

Although the high cost and slow speed make whole-genome sequencing difficult, both limitations are rapidly diminishing, Goldstein says. "A lot of this story is going to be told over the next two or three years." -S.D.

response have evolved. "The exciting thing for me is that more and more people are becoming interested in the host side of this," says Haynes. "We're going to have to understand this area of biology before [an HIV] vaccine is going to be made."

Sarah DeWeerdt is a freelance writer in Seattle.

1. Goonetilleke, N. et al. J. Exp. Med. 206, 1253-1272 (2009) 2. Levesque, M. C. et al. PLoS Med. 6, e1000107 (2009).

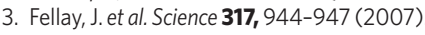

